\title{
Freeway Ramp Metering Using Fuzzy Logic and Genetic Proportional plus Integral Control
}

\author{
Xinrong Liang ${ }^{1,2}$, Qi Lu ${ }^{1}$, Peiqun $\operatorname{Lin}^{2}$ and Jianmin $\mathrm{Xu}^{2}$ \\ ${ }^{1}$ College of Information Engineering, Wuyi University, Jiangmen, China \\ ${ }^{2}$ College of Civil Engineering and Transportation, South China University of \\ Technology, Guangzhou, China \\ xrliang55@126.com
}

\begin{abstract}
Past research has produced various ramp metering approaches. Even so, the results of existing ramp control techniques are unsatisfactory due to the highly nonlinear property of the ramp control system. For improving the ramp metering performance and raising the ramp control accuracy, we propose a mixed approach of fuzzy logic control and proportional plus integral (PI) control for freeway ramp metering. We also employ a genetic algorithm $(G A)$ to optimize the PI parameters in this paper. Furthermore, we apply a nonlinear control technique with a feedback loop to enhance system performance. Firstly, a first-order traffic flow model called hydrodynamic model is established, and the model characteristics are analyzed. Secondly, the control objective of the ramp metering system is defined by the mainline traffic density. Thirdly, a mixed controller of fuzzy logic and genetic PI is designed based upon this hydrodynamic model and in combination with a nonlinear control technique. The membership functions of the fuzzy logic control are triangular or Gaussian curves, and the numbers of fuzzy control rules are nine. Fourthly, optimization procedures of a GA for finding the best PI control parameters are given with details. Finally, the authenticity and effectiveness of the mixed controller are verified with Matlab software R2010a and also by VISSIM microscopic traffic simulation. Simulation results prove that this mixed control approach has better tracking performance and smaller density errors compared with the method of artificial neural networks. This mixed control approach, as well as the nonlinear control technique, provides a new idea for freeway ramp metering.
\end{abstract}

Keywords: Highway Traffic Control, Nonlinear Control Technique, First-Order Model, Fuzzy Logic Control, Genetic Algorithm

\section{Introduction}

China has constructed vast amounts of freeways since eighty years of the last century. Nevertheless, a sharp increase in vehicle numbers and traffic volumes has resulted in severe congestion on freeways. Thus, freeway traffic control has become more and more important. Ramp metering is an important component of freeway traffic control, and it provides high efficient traffic control and management [1]. The chief aim of ramp metering is to regulate the number of vehicles entering into freeway mainlines from onramps so as to take full advantage of freeway resources. Past research has produced various ramp metering approaches, and there exist some useful methods for absorbing and learning. These methods include mathematical programming [2]; decentralized control [3]; proportional-integral-differential control [4]; backstepping control [5]; feedback control [6]. Moreover, these methods also include intelligent control and optimization techniques, such as neural networks [7-9]; optimal control [10-12]; iterative learning control [13]; fuzzy control [14]; cerebellar model articulation control [15]; cellular automaton model [16]; coordinated ramp metering algorithm [17]. We can classify those 
metering algorithms into two categories. One is the fixed-time metering, and the other is the traffic responsive metering. Metering rates in traffic responsive metering are determined based on real-time traffic status. In contrast, the fixed-time metering cannot respond to current traffic conditions. Consequently, traffic responsive metering is superior to the fixed-time metering in improving the freeway efficiency and in eliminating jams. Nowadays, intelligent control techniques are widely applied in the traffic responsive metering. As an intelligent control technique, artificial neural networks are mostly used in freeway traffic control. However, neural networks have some shortcomings in applying to freeway ramp metering. For instance, the training procedures, as well as the training data, have an influence on the network performance. Besides, the system parameters, such as the number of learning epochs and the number of neurons in hidden layers, also exert a tremendous influence on the network performance.

Ramp metering system is an extremely nonlinear control system. For this reason, we use a nonlinear feedback control technique to design the traffic responsive metering algorithm so that we can improve the system performance. For raising the ramp control accuracy, we present a mixed approach of fuzzy logic control and PI control for freeway ramp metering. Also, we employ a GA to optimize the PI parameters. Because fuzzy logic control can easily construct nonlinear controllers, it is thought to be a promising method in many engineering fields. Fuzzy logic control has its rationality since its control strategies are similar to the operational process of experienced operators. Some rules of conditions and actions can state this working process. The conditions use linguistic terms like small or big, and the action representation uses linguistic terms like decrease marginally. Similarly, the fuzzy logic control uses if-then rules. However, fuzzy logic control has an equivalent effect of proportional plus differential (PD) control, and its steady-state deviation generated by disturbance signals may be not zero. Hence, we add an integral control to remove this offset. This mixed control approach combined with a nonlinear feedback control technique has several advantages. First, it can directly deal with a nonlinear problem and needs not to linearize the first-order traffic flow model. Second, GA can online adjust PI control parameters so that the system can adapt to constantly changing environments. Third, it has excellent tracking performance and tiny density errors.

\section{Freeway Hydrodynamic Model}

Lighthill, Whitham, and Richards presented a first-order traffic flow model called the LWR model. The LWR model is a hydrodynamic model with a nonlinear relationship between traffic flow and traffic density. Figure 1 shows a freeway segment, which has multiple lanes in its mainline and only one lane in its entrance ramp. We assume that the flows at time $t$ from the upper boundary and the lower boundary are $Q_{\mathrm{u}}(t) \mathrm{veh} / \mathrm{h} / \mathrm{lane}$ and $Q(t) \mathrm{veh} / \mathrm{h} / \mathrm{lane}$ respectively. We also assume that the flow at time $t$ from the entrance ramp is $R(t) \mathrm{veh} / \mathrm{h}$. According to the vehicular conservation principle, we obtain the equation (1).

$$
n(t+1)=n(t)+\Delta T\left[\lambda Q_{\mathrm{u}}(t)-\lambda Q(t)+R(t)\right]
$$

Here, $n(t)$ is the vehicle numbers in the mainline of this segment at time $t$. Variable $\Delta T$ stands for the sampling interval, and $\lambda$ represents the number of lanes on the freeway mainline.

Dividing by $\lambda L$ into both sides of the equation (1) and defining traffic density by $P(t)=n(t) /(\lambda L)$, we obtain the following density equation.

$$
P(t+1)=P(t)+(\Delta T / L)\left[Q_{\mathrm{u}}(t)-Q(t)+R(t) / \lambda\right]
$$




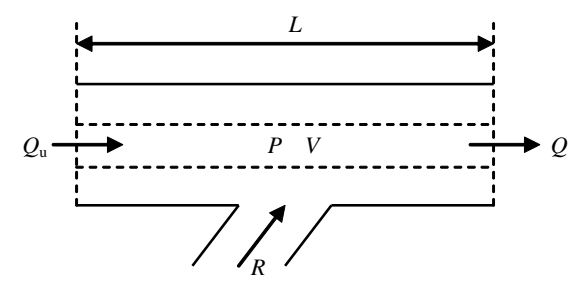

Figure 1. A Freeway Segment

According to the fundamental diagram theory of freeway traffic flow, there exists a function relation between traffic density $P(t)$ and traffic flow $Q(t)$. This feature relation has various forms, such as a triangular shape or a parabolic form. For instance, Newell presented a triangular relationship between flow and density.

$$
Q= \begin{cases}V_{\mathrm{f}} P, & \text { if } P \leq P_{\mathrm{c}} \\ C_{0}\left(P_{\mathrm{jam}}-P\right), & \text { if } P \geq P_{\mathrm{c}}\end{cases}
$$

Here, $V_{\mathrm{f}}$ is the free flow speed, and $C_{0}$ denotes the wave speed propagating in opposition to the traffic stream. Variable $P_{\mathrm{c}}$ is the critical density, and $P_{\text {jam }}$ stands for the jam density.

Green-shield put forward a parabolic relationship between flow and density

$$
Q=V_{\mathrm{f}}\left(P-P^{2} / P_{\mathrm{jam}}\right)
$$

Kotsialos suggested an exponential curve of flow and density.

$$
Q=V_{\mathrm{f}} P\left[1-\left(P / P_{\mathrm{jam}}\right)^{l}\right]^{m}
$$

Here, $l$ and $m$ are real numbers.

These equations of flow and density have several common characteristics described in the following.

(1) There is a zero density point at which flow also equals zero.

(2) There exists a maximal density called a jam density $\left(P_{\text {jam }}\right)$ at which flow is equal to zero.

(3) There is a critical density $\left(P_{\mathrm{c}}\right)$ at which the flow reaches its maximal value.

(4) When the density is less than the critical density, flow increases with the rise of density, and this area is called the uncongested region.

(5) When the density is greater than the critical density, flow decreases as the density increases, and this area is called the congested region.

Hydrodynamic models consist of first-order models and higher-order models according to modeling methods of the mean speed. First-order models employ only the density equation to describe the evolution process of freeway traffic flow because the density uniquely determines the average speed. That is to say, $V=V(P)$ and $Q=P V$. In contrast, higher-order models use the other speed equation, as well as the density equation, for traffic flow modeling.

A commonly used first-order hydrodynamic model includes an equation of flow versus density and the density equation, such as the equation (2). This first-order model has several advantages. First, it offers a simple and practical method for studying the freeway traffic flow. Second, it is suitable for describing many traffic phenomena, such as stop-and-go traffic at bottlenecks. Third, its simulation results are identical to those of VISSIM microscopic traffic simulation. (6).

Defining $\beta=\Delta T / L$ and substituting the equation (4) into (2), we get the equation

$$
P(t+1)=P(t)+\beta Q_{\mathrm{u}}(t)-\beta V_{\mathrm{f}}\left[P(t)-P^{2}(t) / P_{\mathrm{jam}}\right]+\beta R(t) / \lambda
$$


Equation (6) can completely describe the evolution process of the traffic stream in freeway mainlines.

\section{Control Objective of the Ramp Metering System}

We use a ramp control method to keep the freeway in the best situation so that we can take full advantage of freeway facilities. According to the fundamental diagram theory of traffic flow, the flow capacity of a freeway has its limitation. This restriction indicates the desired aim to attain. Because there is a corresponding relation between the density and flow in congested or uncongested regions, the equivalent aim is to keep the density in the vicinity of the critical density. Consequently, the control objective of the ramp metering system is to find a suitable ramp metering rate $R(t)$ so that the actual density $P(t)$ can track the desired density $P_{\mathrm{d}}$. Here, $P_{\mathrm{d}}$ is within the range of $P_{\mathrm{c}}-\delta$ to $P_{\mathrm{c}}+\delta$ in the steady state, and $\delta$ is a real number. As the ramp metering system is an extremely nonlinear system, such a ramp metering rate $R(t)$ cannot be obtained directly from the first-order hydrodynamic model. For this reason, we employ a mixed approach of fuzzy logic control and PI control for generating the $R(t)$ so as to achieve the control objective. Moreover, we employ a GA to optimize the PI parameters so that the system performance is the best.

\section{Traffic Density Controller Based on Fuzzy Logic Control and Genetic PI Control}

\subsection{Combination Structure and Block Diagram of the Feedback Loop plus the Intelligent Control}

Aimed at the nonlinear control problem of the freeway ramp metering, an efficient and practical measure is to introduce feedback control techniques for eliminating traffic noises and disturbance signals, as well as for reducing the modeling errors. As is known to all, the ALINEA presented by Papageorgiou in 1991 is a feedback control method. However, the results of the mixed method of feedback control and traditional control are not satisfactory. Hence, we combine feedback control techniques with intelligent control algorithms. In this way, we can retain the advantages of feedback control technologies, such as the elimination of serious noise disturbance and improvement of the system robustness. At the same time, we can enjoy further performance improvements from intelligent control algorithms, such as excellent dynamic tracking performance and small steady state errors. The smart control algorithms in this paper are hybrid algorithms of fuzzy logic control and genetic PI control. Figure 2 illustrates the combination structure and block diagram of the feedback loop plus the intelligent control.

In Figure 2, $E(t)$ is a density error signal generated by the desired density $P_{\mathrm{d}}$ and the actual density $P(t) . \Delta E(t)$ denotes the density error increment, and $\Delta R(t)$ stands for the metering rate increment. The traffic flow model in Figure 2 is the first-order hydrodynamic model described in Chapter 2. Fuzzy logic control and genetic PI control act on the density error and its increment to generate the ramp metering rate $R(t) . R(t)$ is the control signal sent to the freeway traffic flow model for producing $P(t+1)$. Using this nonlinear feedback control, the actual density $P(t)$ can follow the trajectory of desired density $P_{\mathrm{d}}$. Here, $R(t)$ is a control variable according to the automatic control terminology. $P(t+1)$ is an output variable, and $P(t)$ is a state variable. 


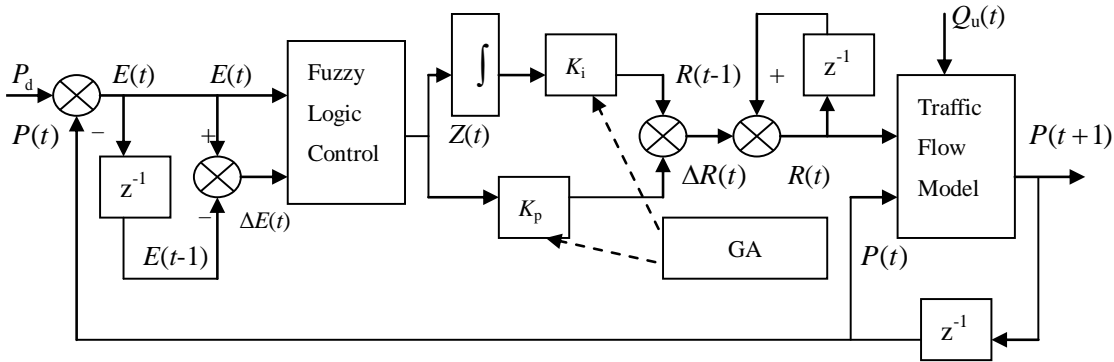

\section{Figure 2. Combination Structure and Block Diagram of the Feedback Loop plus the Intelligent Control}

\subsection{Basic Principle and Design Process of Fuzzy Logic Control}

Figure 3 shows the structure and components of fuzzy logic control. A fuzzy logic controller consists of fuzzification, fuzzy rule, an inference process, and defuzzification. The fuzzification converts each of the input signals into several fuzzy classes that have appropriate linguistic values and membership functions. The fuzzy rule is laid down according to human experience and expert suggestions, and the fuzzy logic control uses if-then rules. These rules have corresponding weight values based on their importance and reliability. According to the fuzzy rules, the inference process is to carry out a comprehensive analysis and evaluation of the conditions of input variables so as to obtain an output variable with linguistic terms. As an inference system includes multiple rules, and these rules have overlapping conditions, one input of the inference system tends to match several fuzzy rules. For this reason, the system needs an approach to superimpose the inference conclusions of all related fuzzy rules. In this paper, we choose Mamdani inference and describe it below.

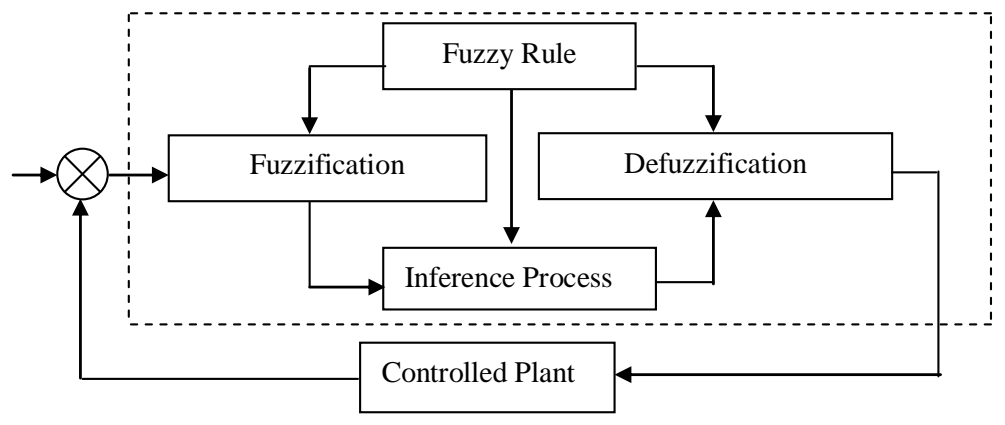

Figure 3. Structure and Components of Fuzzy Logic Control

Suppose there exists a fuzzy rule $r_{i}$.

If (variable $X$ belongs to the language value $A_{i}$ ) and (variable $Y$ belongs to the language value $B_{i}$ ) then (variable $Z$ belongs to the linguistic value $C_{i}$ ), then there is inference intensity $\alpha_{i}$ for the premise of this fuzzy rule.

$$
\alpha_{i}=u_{A_{i}}(X) \wedge u_{B_{i}}(Y)
$$

In the equation (7), $u_{A_{i}}$ or $u_{B_{i}}$ denotes the membership function of $A_{i}$ or $B_{i}$, and $\wedge$ is the minimum operator. The corresponding amount of control of this fuzzy rule is $C_{i}^{\prime}$, and its membership function is $u_{C_{i}}(\mathrm{Z})$.

$$
u_{C_{i}}(Z)=\alpha_{i} \wedge u_{C_{i}}(Z)
$$

In the equation (8), $u_{C_{i}}(\mathrm{Z})$ denotes the membership function of $C_{i}$. 
For a set of fuzzy rules, the final amount of control of all rules is $C$, and $u_{C}(\mathrm{Z})$ is its membership function.

$$
u_{C}(Z)=\underset{i}{\vee}\left(\alpha_{i} \wedge u_{C_{i}}(Z)\right)
$$

Here, $v$ is the maximum operator.

Defuzzification changes the fuzzy result into an exact output value. Commonly used defuzzification methods include the weighted mean method and the center of gravity method. In this paper, we use the center of gravity method to suppress the parameter variations and to overcome the random disturbances.

One output and two inputs of the fuzzy logic control are $Z(t), E(t)$, and $\Delta E(t)$ respectively. Below is the calculation formulas of $E(t)$ and $\Delta E(t)$.

$$
\begin{gathered}
E(t)=P_{\mathrm{d}}-P(t) \\
\Delta E(t)=E(t)-E(t-1)
\end{gathered}
$$

The membership functions of $E(t), \Delta E(t)$, and $Z(t)$ are triangular curves described by linguistic terms $\mathrm{n}$ (negative), $\mathrm{m}$ (medium), and $\mathrm{p}$ (positive), as shown in Figures 4, 5, and 6. $E(t)$ and $\Delta E(t)$ range from -40 to $+40 \mathrm{veh} / \mathrm{km} / \mathrm{lane}$, but $Z(t)$ from -100 to $+100 \mathrm{veh} / \mathrm{h}$.

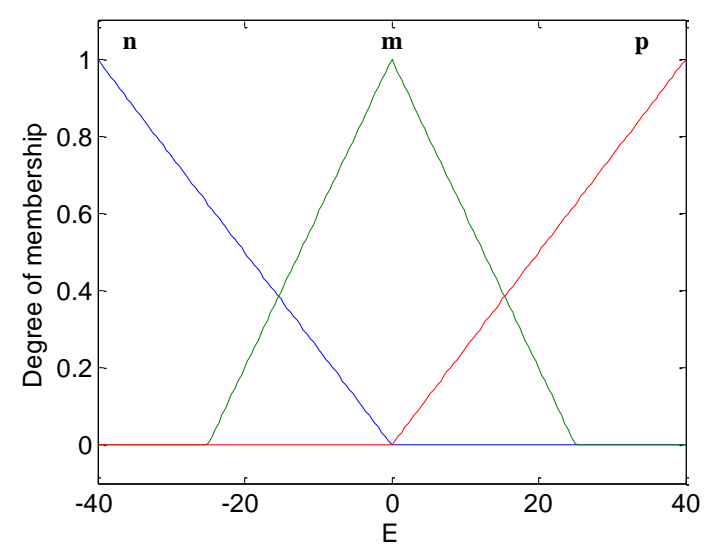

Figure 4. Membership Functions of $E(t)$

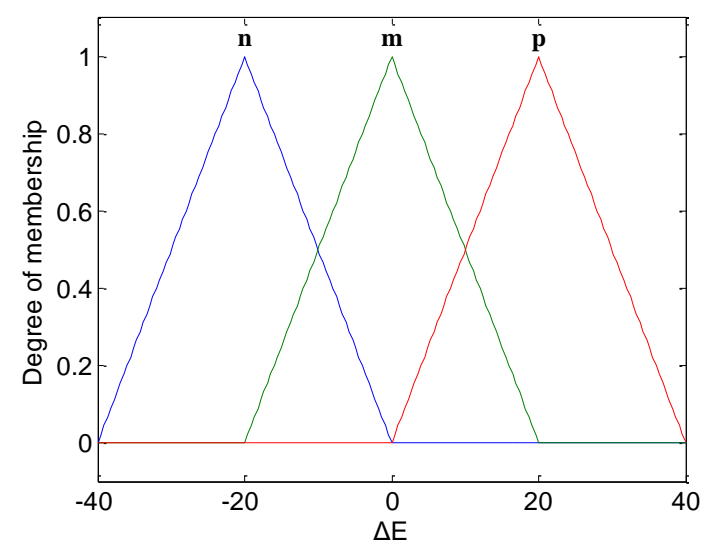

Figure 5. Membership Functions of $\Delta E(t)$ 


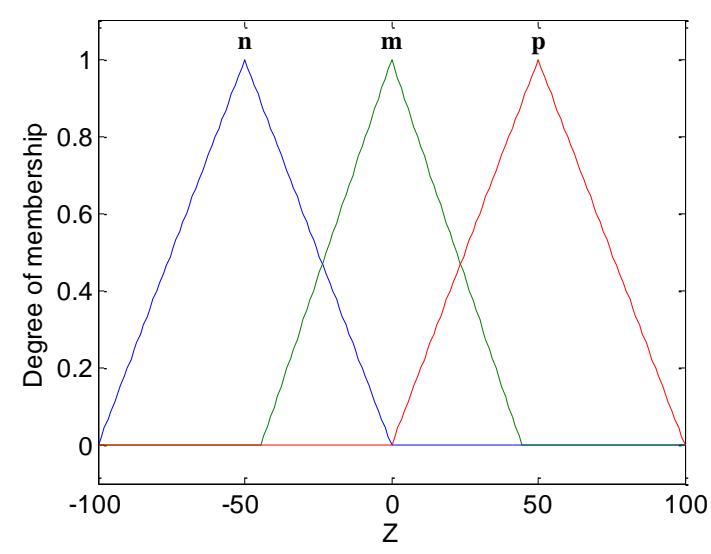

Figure 6. Membership Functions of $Z(t)$

Nine fuzzy rules designed in this paper are as follows.

(1) If ( $E$ is n) and ( $\Delta E$ is n) then ( $Z$ is n).

(2) If ( $E$ is $\mathrm{n})$ and ( $\Delta E$ is $\mathrm{m})$ then $(Z$ is $\mathrm{n})$.

(3) If ( $E$ is $n)$ and ( $\Delta E$ is p) then ( $Z$ is $\mathrm{m}$ ).

(4) If ( $E$ is $\mathrm{m})$ and ( $\Delta E$ is $\mathrm{n})$ then ( $Z$ is $\mathrm{n})$.

(5) If ( $E$ is $\mathrm{m})$ and ( $\Delta E$ is $\mathrm{m})$ then $(Z$ is $\mathrm{m})$.

(6) If ( $E$ is $\mathrm{m})$ and ( $\Delta E$ is p) then ( $Z$ is p).

(7) If ( $E$ is p) and ( $\Delta E$ is $\mathrm{n})$ then ( $Z$ is $\mathrm{m}$ ).

(8) If ( $E$ is p) and ( $\Delta E$ is $\mathrm{m})$ then ( $Z$ is p).

(9) If ( $E$ is p) and ( $\Delta E$ is p) then ( $Z$ is p).

The reason for the last rule is as below. In the final rule, $E(t)$ and $\Delta E(t)$ are positive. In the light of equations (10) and (11), $P(t)$ is smaller than $P_{\mathrm{d}}$, and $P(t)$ is also smaller than $P(t-1)$. That is to say, there exists a downward trend of the actual density. Based on the human experience, we have to increase $Z(t)$. This increase of $Z(t)$ means that $Z(t)$ is positive. In a similar way, we can also explain the reasons for other eight fuzzy rules.

In the process of defuzzification, we calculate the output $Z(t)$ of the fuzzy logic control based on the center of gravity method. The calculation formula for $Z(t)$ is as follows.

$$
Z(t)=\sum_{i=1}^{9}\left(Z_{i}(t) u_{U_{i}}(Z)\right) / \sum_{i=1}^{9} u_{U_{i}}(Z)
$$

In the equation (12), $Z_{i}(t)$ denotes the area center of the membership function of $U_{i}$, and subscript $i$ means the $i^{\text {th }}$ rule. Variable $u$ stands for the membership function value of $U_{i}$, and subscript $U_{i}$ represents the linguistic input of the fuzzy logic control in the $i^{\text {th }}$ rule.

\subsection{Design of Proportional plus Integral Control}

We have developed a controller based on fuzzy logic inference so far. However, this fuzzy logic control is analogous to the proportional plus differential (PD) control, and PD control is unable to eliminate the steady state error. Thus, we add an integral unit to raise the control accuracy and to improve the system performance.

The metering rate increment $\Delta R(t)$ is calculated by PI control.

$$
\Delta R(t)=K_{\mathrm{p}} Z(t)+K_{\mathrm{i}} \sum Z(t)
$$

Here, $K_{\mathrm{p}}$ is the proportional factor, and $K_{\mathrm{i}}$ is the integral factor. $Z(t)$ denotes the output value of the fuzzy logic control, as seen in Figure 2. 


\subsection{Optimization Procedure of the PI Control Parameters with GA}

Genetic algorithm (GA) is a kind of intelligent search technique by the natural selection theory. The data structure used in the GA is similar to the chromosome structure, and the chromosomes are, in fact, a set of strings with an encoding function. GA employs the selection operator, the crossover operator, and the mutation operator to evolve the chromosomes. This evolutionary process usually begins with a group of stochastically produced chromosomes that stand for all the candidate solutions. We encode the various positions of a chromosome into bits or characters, and these bits or characters are called genes. Also, we judge the goodness of a chromosome by an evaluation function and call the evaluation function as a fitness function.

GA consists of five components: the population of individuals, the fitness function, the selection operation, the crossover operation, and the mutation operation. Below lists optimization steps of the GA.

(1) Determine the coding mode of optimized parameters

Optimized parameters are the proportional and integral factors of the PI controller, and the coding mode is the real number coding or binary coding.

(2) Generate the initial population

The initial population stands for the gene coding of PI parameters.

(3) Evaluate the fitness function value

We convert each chromosome into PI parameters whose performance index is judged by its fitness function value. The aim is to minimize the errors between the actual density and the desired density as quickly as possible. Thus, we define a performance index based on the density tracking errors.

$$
J=\sum E^{2}(t)=\sum\left[P_{\mathrm{d}}-P(t)\right]^{2}
$$

Because of a big value of $J$ corresponding to a small fitness function, we define the fitness function as the reciprocal of $J$.

(4) Selection operation

Selection operation is a process that randomly chooses the individual on the basis of its fitness value. A bigger fitness value means that the individual has a higher probability to enter into the next generation. Besides the traditional selection operation, we also use an elitism selection that directly retains the best individual without modification. Elitism selection is unable to lose the optimum PI parameters.

(5) Crossover operation

Crossover operation is used to improve the global searching ability of the GA, which generates the new generation by combining two parent values. In this paper, we use a gene recombination approach for producing individuals.

(6) Mutation operation

Mutation operation is utilized to add new genetic information to the population so as to increase the diversity of species further. The mutation process for real variables is to generate random numbers and add them to the variables with a given small probability value. In this paper, the mutation probability dynamically changes with the fitness value, and a bigger fitness value corresponds to a smaller mutation probability.

We repeat the above steps until reaching the specified number of iterations or until satisfying the optimization criteria. In the end, we obtain the optimal PI parameters by the GA. 


\section{Simulation Results}

We simulate the traffic density controller based on fuzzy logic control and genetic PI control shown in Figure 2 with Matlab software. The traffic flow model in Figure 2 is the first-order hydrodynamic model described in Chapter 2. These model parameters are as follows. Free flow speed $V_{\mathrm{f}}=97.3 \mathrm{~km} / \mathrm{h}$, critical density $P_{\mathrm{c}}=42 \mathrm{veh} / \mathrm{km} / \mathrm{lane}$, and jam density $P_{\text {jam }}=84 \mathrm{veh} / \mathrm{km} / \mathrm{lane}$. The flow capacity $Q_{\mathrm{m}}=2043.3 \mathrm{veh} / \mathrm{h} / \mathrm{lane}$. The number of lanes on the freeway mainline is equal to 3 . Sampling interval $\Delta T=20 \mathrm{~s}$, and the desired density $P_{\mathrm{d}}=39.1 \mathrm{veh} / \mathrm{km} / \mathrm{lane}$.

Figure 7 shows the flow curves of the upper boundary. Figure 8 illustrates the desired density in the freeway mainline. The best PI parameters optimized by the GA are $250.9754\left(K_{\mathrm{p}}\right)$ and $105.5590\left(K_{\mathrm{i}}\right)$. Figure 9 shows the actual density in the freeway mainline, and Figure 10 shows the flow from the entrance ramp. Figures 7 , 9 , and 10 indicate that both $P$ and $R$ exist transition points when $Q_{\mathrm{u}}$ is a step function. However, the change time of $P$ or $R$ is very short, and each of them quickly enters into a steady state. Moreover, the steady state value of $P$ is $39.1 \mathrm{veh} / \mathrm{km} / \mathrm{lane}$, which is identical to the desired density $P_{\mathrm{d}}$. When $Q_{\mathrm{u}}$ is 1790 , $1930,1840,1720,1820$, and $1930 \mathrm{veh} / \mathrm{h} / \mathrm{lane}$, the steady state value of $R$ is $730.6752, \quad 310.6753, \quad 580.6753,940.6752,640.6752$, and $310.6753 \mathrm{veh} / \mathrm{h}$ respectively.

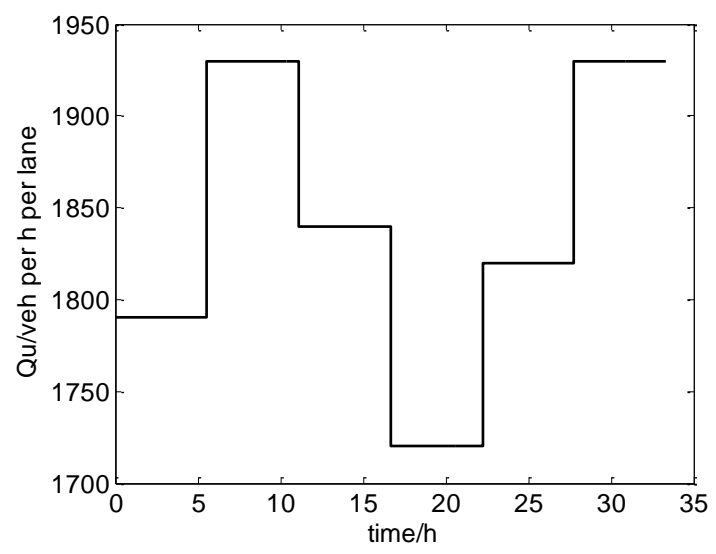

Figure 7. Flow Curves of the Upper Boundary

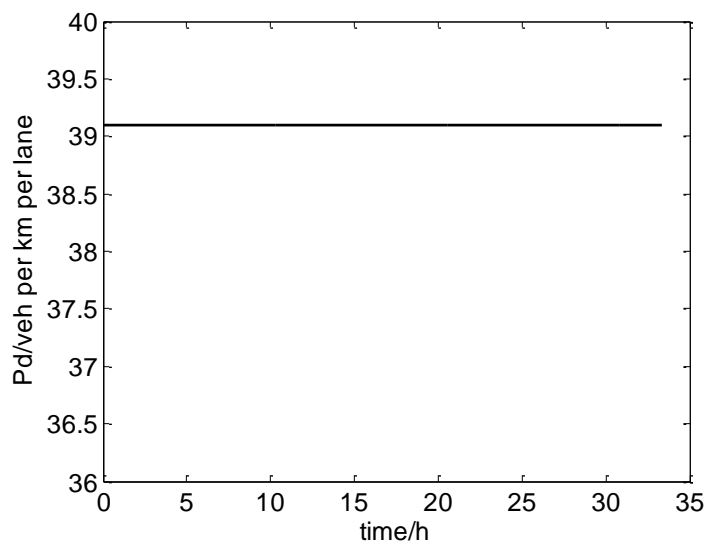

Figure 8. Desired Density in the Freeway Mainline 


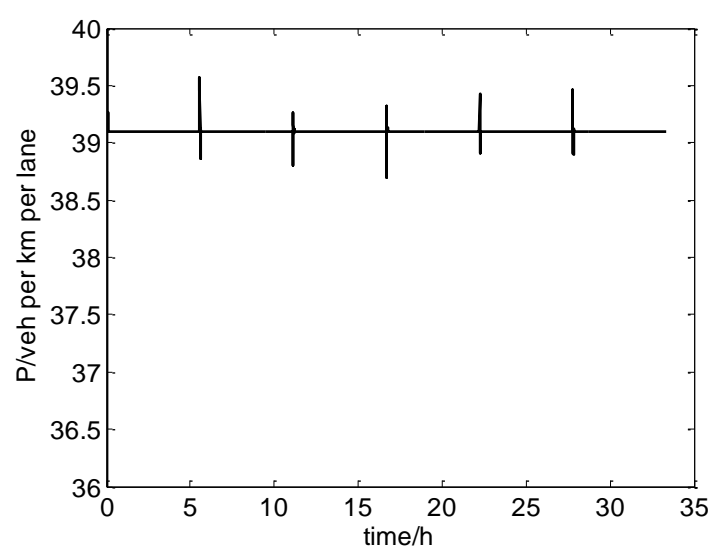

Figure 9. Actual Density in the Freeway Mainline

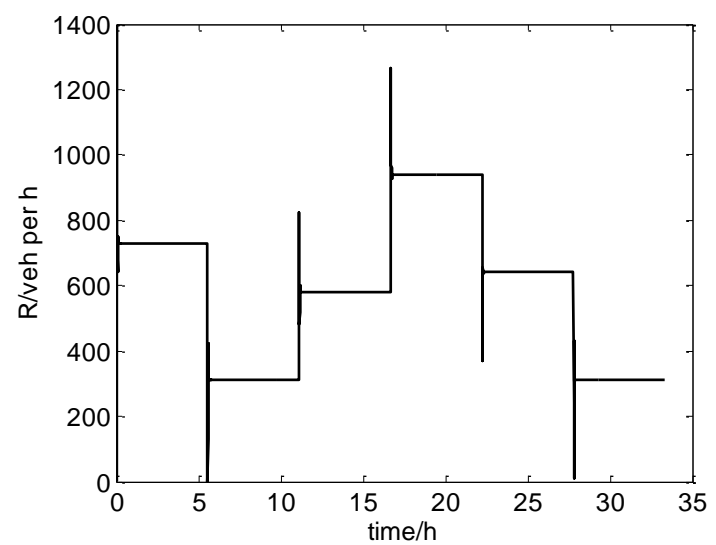

Figure 10. Flow from the Entrance Ramp

For further illustrating the properties of the mixed control method proposed in this paper, we compare the simulation results in this article with those in reference [8]. Literature [8] combines neural networks with the principle of automatic control and designs self-adaptive ramp metering algorithms. Simulation results prove that the neural network method has some advantages. However, from the simulation results in [8] we can see that the tracking performance of the neural network method needs improvement. Also, the neural network method exists tracking errors in the steady state.

Compared with artificial neural networks [8], the mixed control method presented in this paper has better metering performance and higher control accuracy. It has excellent dynamic tracking performance, and its steady state errors are zero. Moreover, the GA can succeed in finding the optimal PI control parameters. Thus, the system performance is improved.

\section{Result Comparison and Test}

\subsection{Results of the Fuzzy Logic Mixed Controller with Gauss Membership Functions}

In Chapter 5, we have simulated the mixed controller shown in Figure 2, and we have chosen triangular membership functions for all the input and output variables of the fuzzy logic control. Triangular membership functions are relatively simple. If we use more complicated Gauss membership functions, how will the results be? In this section, a fuzzy logic mixed controller with Gauss membership functions is 
simulated, and Figure 2 shows the block diagram of this mixed controller. $E(t)$, $\Delta E(t)$, and $Z(t)$ in Figure 2 are described by three linguistic terms $\mathrm{n}$ (negative), $\mathrm{m}$ (medium), and $\mathrm{p}$ (positive). All the input and output variables are Gauss membership functions as shown in Figures 11, 12, and 13. Other simulation conditions in this section are the same as those in Chapter 5.

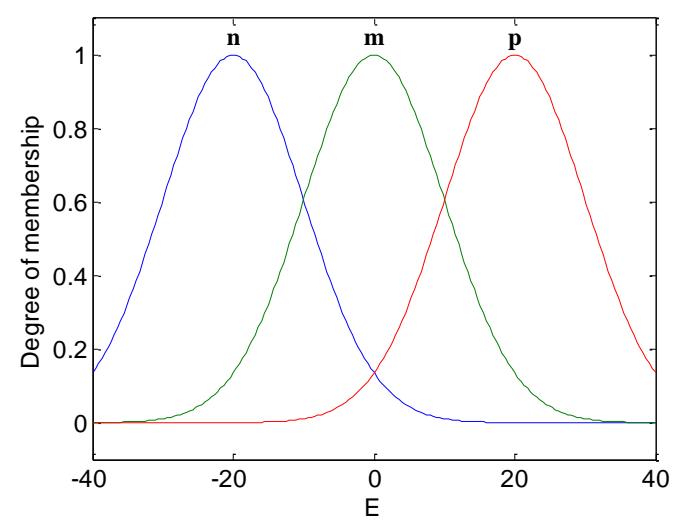

Figure 11. Gauss Membership Functions of $E(t)$

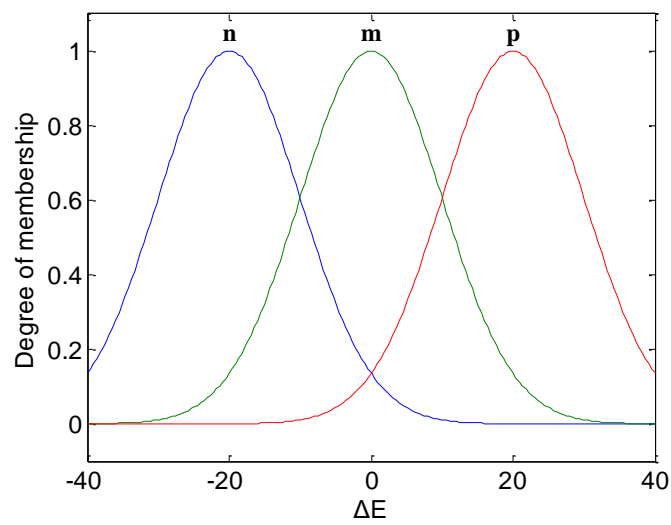

Figure 12. Gauss Membership Functions of $\Delta E(t)$

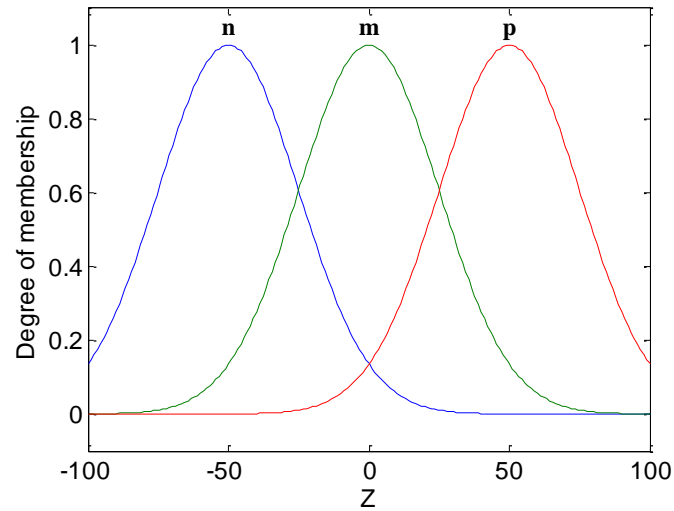

Figure 13. Gauss Membership Functions of $Z(t)$

The actual density in the freeway mainline and flow from the entrance ramp using Gauss membership functions can be obtained by simulation as shown in Figure 14 and Figure 15 respectively. The steady state values of $P$ and $R$ in this section are 
identical to those in Chapter 5. That is to say, the fuzzy logic mixed controller with Gauss membership functions has the same steady state results as those with triangular membership functions.

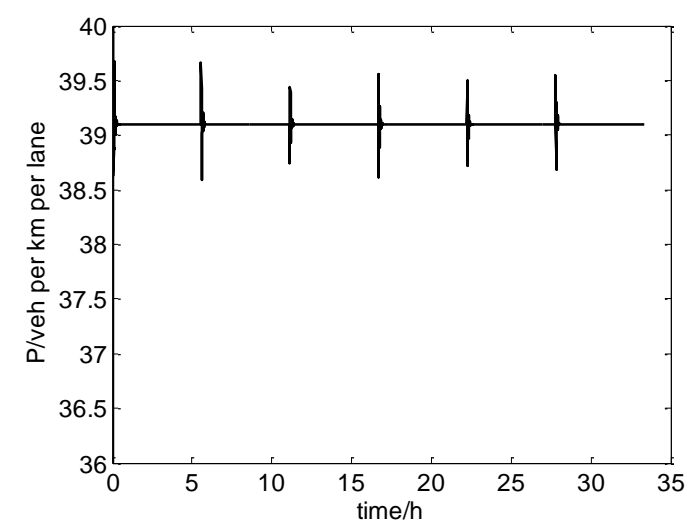

Figure 14. Actual Density in the Freeway Mainline

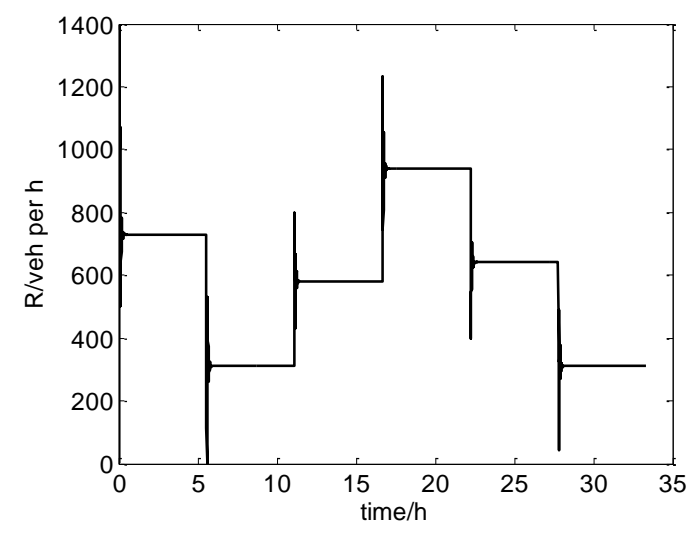

Figure 15. Flow from the Entrance Ramp

\subsection{Testing Results via VISSIM Microscopic Traffic Simulation}

In recent years, microscopic simulation software has been widely used to evaluate the effects of traffic control algorithms. This section conducts testing of the traffic model and control algorithms proposed in this paper via VISSIM microscopic traffic simulation. VISSIM is a microscopic and behavior based model used for simulating freeway traffic and urban traffic, as well as public transportation. Recent experiments show that the ramp metering values generated by the VISSIM are practically no different from those produced by real world controllers. There exist three steps in the procedure for the VISSIM simulation.

(1) Build a VISSIM model.

(2) Determine inputs and outputs of the VISSIM model, and set up simulation parameters.

(3) Conduct simulation evaluations, and obtain simulation results.

After completing the VISSIM simulation, we can get some statistical values of traffic variables. These variables include upstream inflow $Q_{\mathrm{u}}$, downstream flow $Q$, downstream traffic density $P$, downstream speed $V$, ramp metering rate $R$, and ramp queue length. We only list the VISSIM statistical values of $Q_{\mathrm{u}}, P$, and $R$ to compare the simulation results in Chapter 5 with the VISSIM simulation values. In the first period (0-5.5556 hours), the statistical values of $Q_{\mathrm{u}}, P$, and $R$ are $1797 \mathrm{veh} / \mathrm{h} / \mathrm{lane}$, 
$39.88 \mathrm{veh} / \mathrm{km} / \mathrm{lane}$, and $719 \mathrm{veh} / \mathrm{h}$ respectively. In the second period $(5.5556-11.1111$ hours), the statistical values of $Q_{\mathrm{u}}, P$, and $R$ are $1930 \mathrm{veh} / \mathrm{h} /$ lane, $38.75 \mathrm{veh} / \mathrm{km} /$ lane, and $305 \mathrm{veh} / \mathrm{h}$ respectively. In the third period (11.1111-16.6667 hours), the statistical values of $Q_{\mathrm{u}}, P$, and $R$ are $1842 \mathrm{veh} / \mathrm{h} / \mathrm{lane}, 39.04 \mathrm{veh} / \mathrm{km} / \mathrm{lane}$, and $570 \mathrm{veh} / \mathrm{h}$ respectively. In the fourth period (16.6667-22.2222 hours), the statistical values of $Q_{\mathrm{u}}, P$, and $R$ are $1720 \mathrm{veh} / \mathrm{h} / \mathrm{lane}, 38.53 \mathrm{veh} / \mathrm{km} / \mathrm{lane}$, and $951 \mathrm{veh} / \mathrm{h}$ respectively. In the fifth period (22.2222-27.7778 hours), the statistical values of $Q_{\mathrm{u}}, P$, and $R$ are $1819 \mathrm{veh} / \mathrm{h} / \mathrm{lane}, 39.10 \mathrm{veh} / \mathrm{km} / \mathrm{lane}$, and $645 \mathrm{veh} / \mathrm{h}$ respectively. In the sixth period (27.7778-33.3333 hours), the statistical values of $Q_{\mathrm{u}}, P$, and $R$ are $1931 \mathrm{veh} / \mathrm{h} / \mathrm{lane}, 39.18 \mathrm{veh} / \mathrm{km} / \mathrm{lane}$, and $327 \mathrm{veh} / \mathrm{h}$ respectively.

From the results mentioned above, we can see that the VISSIM statistical values are entirely consistent with the simulation results in Chapter 5. Thus, the VISSIM microscopic traffic simulation gives support to the mixed control method proposed in this paper.

\section{Conclusions}

Aiming at the highly nonlinear property of the ramp control system, we present a nonlinear feedback method and employ intelligent control techniques to design traffic responsive metering algorithms in this paper. We expound the ramp metering problem as a density tracking process, and the traffic evolution model is considered to follow the laws described by the LWR model. In the mixed control algorithms, we use fuzzy logic control and genetic PI control to handle the nonlinear system directly without linearization treatment. The GA is applied to tune the PI parameters so that the control system can adapt to changing circumstances. Simulation results confirm the validity of the nonlinear intelligent control techniques and obtain good response curves.

\section{Acknowledgments}

This work was supported by Guangdong Natural Science Foundation (No. S2012010010356) and by characteristics \& innovation project of Guangdong (No. 2014KTSCX132).

\section{References}

[1] M. Papageorgiou and A. Kotsialos, "Freeway Ramp Metering: an Overview", IEEE Trans. on Intelligent Transportation Systems, vol. 3, no. 4, (2002), pp. 271-281.

[2] M. Papageorgiou, "A New Approach to Time-of-Day Control Based on a Dynamic Freeway Traffic Model”, Transportation Research Part B, vol. 14, no. 4, (1980), pp. 349-360.

[3] N. B. Goldstein and K. S. P. Kumar, "A Decentralized Control Strategy for Freeway Regulation", Transportation Research Part B, vol. 16, no. 4, (1982), pp. 279-290.

[4] Y. Y. Liu, X. R. Liang and T. Huang, "PID Ramp Controller Regulated by Radial Basis Function Neural Network", Proceedings of the 2nd International Conference on Computer Science and Network Technology, Changchun, China, December 1771-1775, (2012).

[5] C. C. Chien, Y. P. Zhang and P. A. Ioannou, "Traffic Density Control for Automated Highway Systems", Automatica, vol. 33, no. 7, (1997), pp. 1273-1285.

[6] T. H. Chang and Z. Y. Li, "Optimization of Mainline Traffic via an Adaptive Coordinated RampMetering Control Model with Dynamic OD Estimation", Transportation Research Part C, vol. 10, no. 2, (2002), pp. 99-120.

[7] H. M. Zhang, S. G. Ritchie and R. Jayakrishnan, "Coordinated Traffic-Responsive Ramp Control via Nonlinear State Feedback”, Transportation Research Part C, vol. 9, no. 5, (2001), pp. 337-352.

[8] H. M. Zhang and S. G. Ritchie, "Freeway Ramp Metering Using Artificial Neural Networks", Transportation Research Part C, vol. 5, no. 5, (1997), pp. 273-286.

[9] A. D. Febbraro, T. Parisini, S. Sacone and R. Zoppoli, "Neural Approximations for Feedback Optimal Control of Freeway Systems", IEEE Trans. on Vehicular Technology, vol. 50, no. 1, (2001), pp. 302313.

[10] A. Alessandri, A. D. Febbraro, A. Ferrara and E. Punta, "Optimal Control of Freeways via Speed Signalling and Ramp Metering”, Control Engineering Practice, vol. 6, no. 6, (1998), pp. 771-780. 
[11] H. M. Zhang and W. W. Recker, "On Optimal Freeway Ramp Control Policies for Congested Traffic Corridors", Transportation Research Part B, vol. 33, no. 6, (1999), pp. 417-436.

[12] A. Kotsialos, M. Papageorgiou, M. Mangeas and H. Haj-Salem, "Coordinated and Integrated Control of Motorway Networks via Nonlinear Optimal Control", Transportation Research Part C, vol. 10, no. 1, (2002), pp. 65-84.

[13] Z. S. Hou, J. X. Xu and J. W. Yan, "An Iterative Learning Approach for Density Control of Freeway Traffic Flow via Ramp Metering”, Transportation Research Part C, vol. 16, no. 1, (2008), pp. 71-97.

[14] X. R. Liang, Z. Y. Liu and Z. Y. Mao, "Nonlinear Feedback Ramp Controller in Freeway Based on Fuzzy Logic", Control Theory \& Applications, vol. 23, no. 1, (2006), pp. 99-102.

[15] X. R. Liang, Y. Y. Liu, G. Y. Man and J. M. Xu, "Density Control for Freeway Traffic Flow Based on the Composite of PID Controller and Cerebella Model Articulation Controller", Control Theory \& Applications, vol. 30, no. 10, (2013), pp. 1281-1286.

[16] C. Q. Mei, H. J. Huang and T. Q. Tang, "A Cellular Automaton Model for Studying the on-Ramp Control of Highway”, Acta Physica Sinica, vol. 57, no. 8, (2008), pp. 4786-4793.

[17] W. Sarintorn, "Development and Comparative Evaluation of Ramp Metering Algorithms Using Microscopic Traffic Simulation", Journal of Transportation Systems Engineering and Information Technology, vol. 7, no. 5, (2007), pp. 51-62.
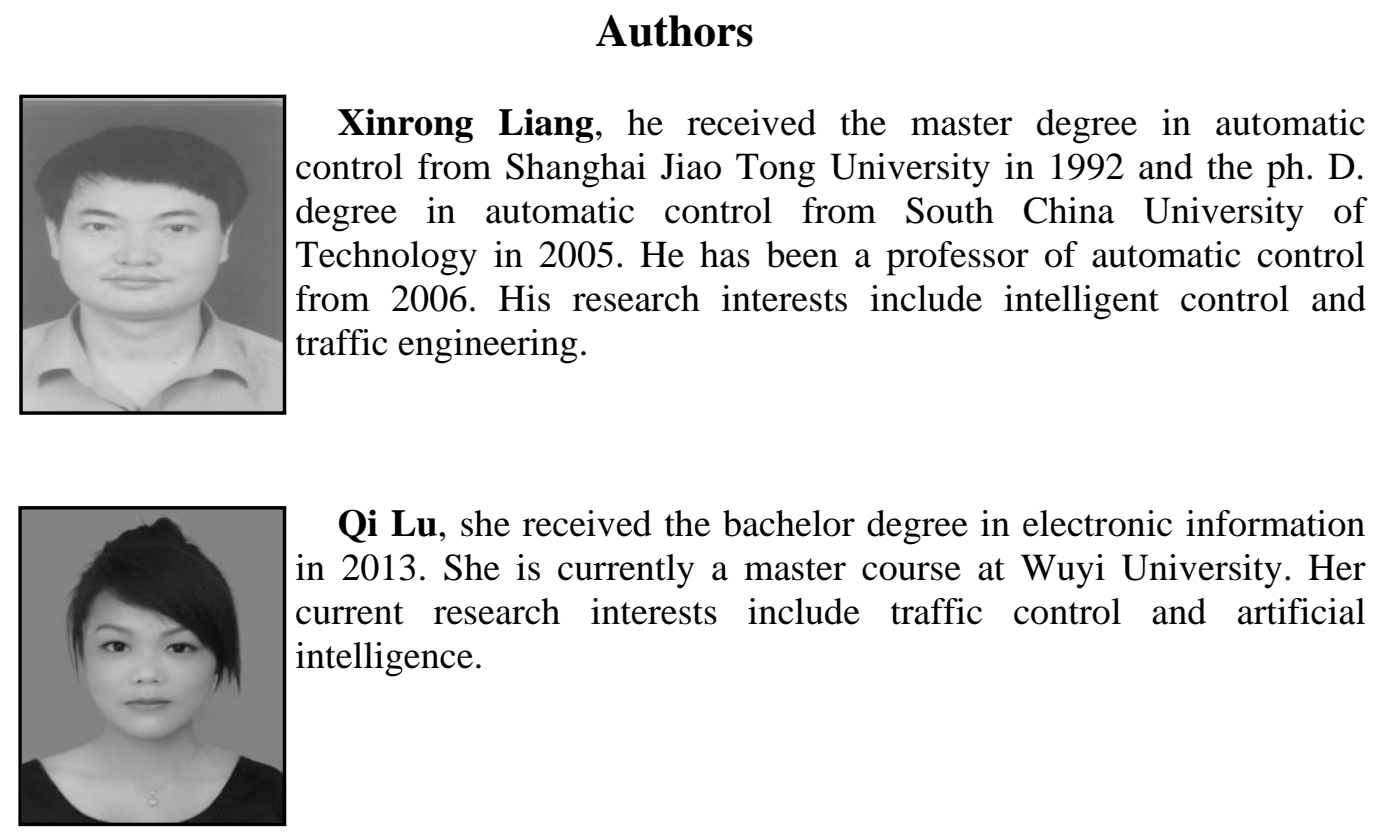

Qi Lu, she received the bachelor degree in electronic information in 2013. She is currently a master course at Wuyi University. Her current research interests include traffic control and artificial intelligence.

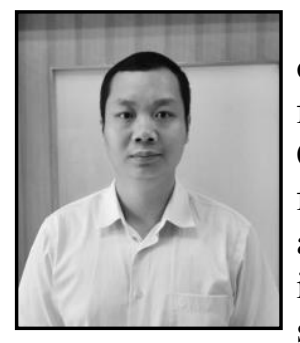

Peiqun Lin, he is an associate professor at South China University of Technology, Guangzhou, China. He received the Ph.D. degree from the School of Automation Science and Engineering at South China University of Technology in 2008. He received his B.S. degree from the School of Transportation and Communication Engineering at South China University of Technology in 2003. His research interests include connected-vehicle network, transportation system simulation, and computer vision and transportation big data analytics. $\mathrm{He}$ has co-authored more than 30 journal publications and more than 15 peer-reviewed conference papers. He holds 13 Chinese patents and has a few patents pending in China. 


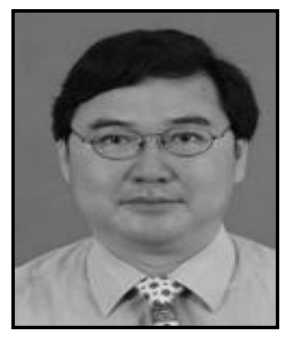

Jianmin $\mathbf{X u}$, he is a professor at South China University of Technology, Guangzhou, China. He received the M.S. and Ph.D. degree from the School of Automation Science and Engineering at South China University of Technology in 1986 and 1994 respectively. He received his B.S. degree from the Department of Electrical Engineering at Jiangxi Institute of Technology in 1982. His research interests include traffic control, transportation system simulation, transportation planning, and transportation big data analytics. He has co-authored more than 100 journal publications and more than 60 peer-reviewed conference papers. He holds 28 Chinese patents and has a few patents pending in China. 
International Journal of Control and Automation Vol. 9, No. 11 (2016) 